\title{
Oferta Académica Flexible bajo el Concepto del Justo a Tiempo
}

\author{
Adalberto G. Díaz \\ Universidad EAFIT, Escuela de Ingenierías, Departamento de Ingeniería de Producción, \\ Apartado Aéreo 3300, Medellín-Colombia (e-mail: gdiaz@eafit.edu.co)
}

\section{Resumen}

Se propone e implementa de un sistema de oferta flexible de los currículos universitarios, como alternativa y solución al mejoramiento de calidad en el aprendizaje de los estudiantes, visualizando la realidad y los prospectos de ejecución para la renovación universitaria. Se discute la operatividad del ofrecimiento flexible como la alternativa estratégica del concepto just in time al servicio de la enseñanza del conocimiento en la educación superior. El sistema opera bajo la modalidad de créditos, lográndose un modelo organizado más abierto y dinámico que permite al estudiante elegir y planear por periodo su desempeño académico, de acuerdo a sus condiciones de estudio y esfuerzo, y también a su entorno social, laboral y académico.

Palabras claves: oferta académica flexible, currículo universitario, crédito académico, justo a tiempo

\section{The Flexible Academic Offering under the Concept of the Just In Time}

\begin{abstract}
A flexible system for the university curriculum offering is proposed and implemented, as an alternative and a solution to the improvement of quality in the learning process of the students. The operability of the flexible offer as the strategic alternative of the concept just in time to the service of higher education is discussed. The system operates under the credits modality, obtaining a organized model more open and dynamic that allows students to choose and plan each period their academic performance, according to their studying conditions and effort, and also to their social, working, and academic environment
\end{abstract}

Keywords: flexible academia offering, university curriculums, academic credit, just in time 


\section{INTRODUCCIÓN}

La educación es un derecho fundamental de la humanidad; es una exigencia y un propósito de todos los gobiernos para ofrecer de un producto académico como un servicio obligatorio del entorno social, el cual exige ser caracterizado en el momento histórico de acuerdo con los arraigos culturales de sus gentes (Diaz, 2002). Por eso, la oferta de un producto académico competitivo y con calidad, exige buscar nuevas alternativas y estrategias para el fortalecimiento de la autoridad académica y el mejor resultado del aprendizaje con técnicas, métodos y modelos de enseñanza aprendizaje actualizados. La observación histórica de los modelos de oferta académica en la universidad puede ubicarse inicialmente a partir de la Revolución Francesa, cuando se engendraron cambios en las concepciones académicas, sociales e industriales, que van más allá del simple desarrollo o de la evolución científica y tecnológica, imponiéndose costumbres de acuerdo con las clases sociales, culturales o las épocas climáticas, tales como: fiestas de temporadas, vacaciones de verano, estilos de vida diferentes, excursiones, indumentarias por ocasión, etc. (Carbajo, 2006). La universidad también se adhiere a la participación de una vida cultural, creándose así el modelo programación académica de la universidad por periodos anuales intercalados con periodos de atención al campo o de vacaciones en verano. La educación universitaria se ha involucrado en la clasificación social volviéndose privilegio de unos y discriminatorio de otros; pero algunos tendrían la oportunidad de lograr una mejor posición en su grupo por contienda en el desempeño como mejor estudiante. Estos modelos han evolucionado a través del tiempo, como también ha crecido el volumen de temas y áreas de estudio, transformándose el mundo de hoy hacia una sociedad del conocimiento, enriquecido y soportado en economías basadas en la información, la tecnología y la sociedad. Pero algunos principios universitarios esenciales siguen vigentes, se ha llegado a creer que el mejor estudiante es el que más materias esté cursando o el más exitoso en las más difíciles. Por su parte también la universidad ha realizado clasificaciones temáticas por áreas del saber, creando dependencias administrativas llamadas facultades y programas de un conjunto de cursos académicos que definen una carrera, a los cuales un estudiante responde con sus intereses y habilidades para obtener un título profesional.

Operativamente, la universidad ha creado un modelo de oferta académica rígido, siendo exitoso en su momento debido al sometimiento a una disciplina extrema del estudiante y sus docentes. Se han establecido métricas del aprendizaje por medio de evaluaciones, pero muchas veces los resultados no reflejan el alcance de los objetivos. De allí que el estudiante aprendió a hacer fraudes en las exámenes, modificando los objetivos del aprendizaje por un mejor nivel de la evaluación a cualquier costo disciplinar o académico. Los currículos universitarios ayudaron en este desenfreno apilando programas con altos volúmenes de contenidos sin preparatorios, muchas veces redundantes y extremadamente difíciles.

En el ocaso del siglo XX llega la conciencia de los grandes cambios con reflexiones como: el conocimiento y el espíritu de formación es un patrimonio de los países y la educación es un tesoro (Delors, 1996), que deben cuidar y capitalizar en resultados tales como índices de crecimientos educativos, índices de desarrollo de tecnologías e investigaciones propias (Diaz, 2002). El alcance de estos objetivos no sólo requiere inversión de capitales, sino de la adopción de estructuras, políticas y métodos de oferta y enseñanza que respondan a las nuevas necesidades creando un sistema de educación superior diferenciado y competitivo a cada estudiante según su productividad (Pedroza-Flores, 2001). Con dinero se pueden construir bibliotecas, edificios y comprar información, pero con estrategias de ofrecimiento y métodos de aprendizaje, este conocimiento permeará las mentes de los hombres y se logrará el desarrollo de los pueblos. Es el reto de pasar de un paradigma centrado en la enseñanza y la transmisión de conocimientos a otro centrado en el aprendizaje (Delors, 1996). También, alrededor de los años 70 los japoneses ven que el desarrollo económico debido a la industrialización empieza a decaer y observan que el reto es la flexibilidad de sus procesos y de la oferta de sus productos, aplicando el modelo del sistema utilizado por Toyota para flexibilizar sus procesos y que posteriormente se conoce como la técnica Justo a Tiempo (Ohno, 1988). Esta técnica ha sido la congruencia del desarrollo industrial, haciendo pensar que la dinámica del mundo es flexible. También los pensadores en el tema de la educación, acuñan el término "educación flexible" (Salinas, 2000) para permitir a una gran 
población de estudiantes moverse en la aldea global del conocimiento. En la Conferencia Mundial sobre Educación Superior celebrada en París en 1998 (Unesco, 1998) se hizo evidente la necesidad de contar con sistemas de educación más diversificados, que ofrezcan una amplia gama de posibilidades de educación y formación mediante nuevos modelos educativos centrados en el estudiante, proyectando el conocimiento como un bien universal (Salinas, 1997), por ello se planea una enseñanza superior caracterizada por su dimensión internacional con movilidad global (Delors, 1996), donde los idiomas extranjeros y sobre todo las nuevas tecnologías de la información y la comunicación sean estrategias fundamentales.

En la publicación "La flexibilización de la oferta académica mejora la calidad del aprendizaje" (Diaz, 2006) se hace referencia al informe de la UNESCO sobre la educación para el siglo XXI (Delors, 1996) señalando que la educación es un proceso permanente, el cual se acomoda a diferentes situaciones del crecimiento continuo del hombre, donde se requiere de un escenario, productos, programas y métodos de enseñanza educativos, que incorporan características de calidad, flexibilidad, diversidad y el desarrollo de competencias transferibles a contextos diferentes de tiempo y espacio, permitiendo y favoreciendo la formación continua de los individuos, la actualización en su disciplina profesional y su crecimiento intelectual, demandando desarrollar la capacidad de aprender a aprender (Nieto,2002).

El termino flexibilizar es contrario a la rigidez y actúa complementariamente, encontrándose delimitado por el entorno sistémico enmarcado por una fuerte normatividad en la cual opera la organización universitaria y que ahora debe mover las fronteras de la rigidez, para dar paso a situaciones cada vez más flexibles, cambiantes e indeterminadas (Casassus, 2000). Es de notar que la formación rígida tiene ventajas indudables, puesto que se orienta a la formación del carácter. Kant en su trabajo sobre pedagogía (Kant, 1803) menciona que "negar la disciplina es un mal mayor que negar la cultura" y Hegel escribió que la disciplina es la columna vertebral del carácter, entonces la disciplina es parte de la formación en la adolescencia, mientras que la formación cultural tiene su espacio durante la vida adulta. Se podría pensar que la formación en la universidad tiene una gran componente de la expansión cultural del hombre, quedando por verificar el momento en la vida del joven en que ocurre este trance de la adolescencia hacia la vida adulta. Lo cierto es que ahora los jóvenes se han hecho más adultos a una edad más temprana, casi que este cambio ocurre durante el periodo que transitan por la universidad, cuando estrenan mayoría de edad con el respectivo derecho de ciudadanía adulta. A su vez los modelos universitarios han evolucionado muy poco en la relación con la descripción de las representaciones y prácticas cotidianas de los estudiantes, con la indiferencia del éxito o el fracaso académico (Leite, 1999), lo cual ha permitido comprender las diversas y múltiples situaciones académicas por las que pasan los estudiantes, además de las condiciones y factores institucionales (Guerra et al., 2002).

\section{ANÁLISIS Y DIAGNÓSTICO A PRINCIPIOS DEL TERCER MILENIO}

En la realidad del funcionar universitario es preocupante la deserción universitaria y el fracaso académico que pueden estar relacionados a razones vocacionales, razones de entorno social o razones de carga académica debida a la distribución del uso del tiempo (McMeekein, 1993). Las políticas de gestión académica orientadas a incrementar el tiempo requerido para el aprendizaje tienen un importante impacto sobre la calidad y la eficiencia en el aprendizaje, pues el uso moderado del tiempo sigue siendo una condición fundamental para la enseñanza efectiva, sin lo cual ningún programa educativo puede avanzar. Cuando los estudiantes se exponen a situaciones adversas constantes, aparecen condiciones afectivas negativas que dificultan el aprendizaje, pues basta ver las consecuencias de una elevada carga académica en ciertos instantes de los periodos de estudio, donde se pone a prueba la disciplina y la capacidad de trabajo del estudiante en límites de exigencia máxima, rompiendo la tan elaborada capacidad disciplinar conseguida durante el tiempo de formación desde la básica. La causa de esta ruptura radica en la forma como se ha concebido el sistema universitario con un modelo rígido, bajo la única alternativa de ofrecimiento de un número de cursos con ejecución simultánea impuestos al estudiante por periodos anuales o semestrales. 
Los modelos pedagógicos en su sistema natural responden a una exigencia ascendente en el tiempo de avance, como se muestra en la Fig. 1, de tal forma que la evaluación del aprendizaje se hace en forma acumulativa hacia el final del curso, llegando a una saturación de trabajo académico con responsabilidad en el estudiante debido al volumen de información, de estudio y de análisis que debe asimilar y hacer para aprobar los cursos, convirtiéndose el proceso de estudio universitario en un aprendizaje traumático, estresante y en un reto violatorio de su integridad individual en momentos de acoso académico. Ante esta situación, la reacción es la respuesta del estudiante a un hecho natural de defensa biológica, lo primero que se da es el cambio de objetivo del proceso académico, se ha cambiado: el aprender un tema por el rescatar una nota a cualquier costo, pues el proceso académico se mide finalmente por el resultado de una nota, la que no necesariamente refleja un real resultado del proceso de aprendizaje en medio de un bajo rendimiento académico manifestado por la pérdida de materias, conocimientos pobres de los contenidos, aumento en la cancelación de cursos, ausentismo frecuente en las clases, fraudes en los exámenes y deserción de las aulas.

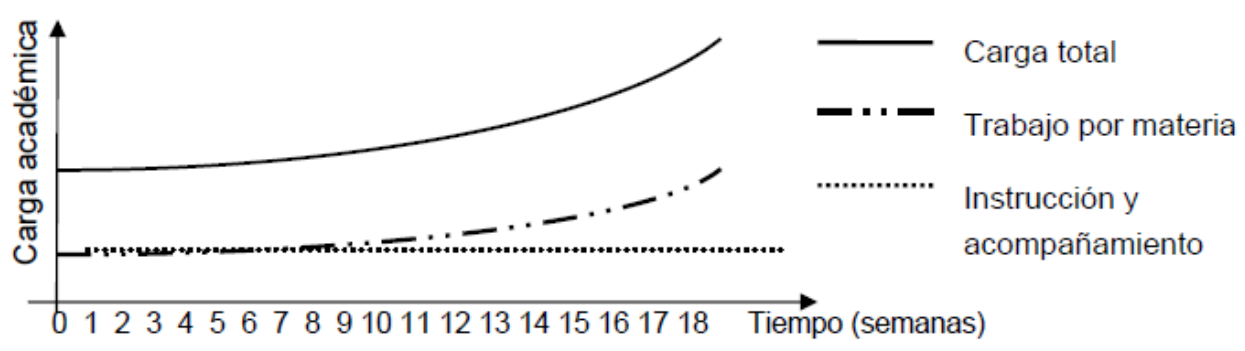

Fig. 1: Distribución de la carga académica del estudiante de acuerdo a las actividades de aprendizaje

El sistema universitario involucra cuatro elementos sistémicos fundamentales: estudiantes, docentes, dirección y entorno. Por consiguiente cualquier mal funcionamiento en uno de estos elementos afecta a todo el sistema. El docente es el custodio del conocimiento y el conductor del proceso de aprendizaje (Delors, 1996), también es el interventor del ambiente que rodea el proceso académico y es por consiguiente el responsable de sugerir o propiciar las condiciones justas para su exitoso desempeño. También es el observador del contraste resultante de su trabajo académico debido a que comparte el tiempo de los estudiantes simultáneamente con colegas de otros cursos y con temáticas diferentes e igual importancia, que también exigen el derecho de un ambiente de desempeño eficaz. El ejercicio docente también requiriere de espacios de acción para la actividad académica, programas de capacitación e investigación, asesorías, periodos sabáticos, pasantías, intercambios, los cuales se encuentran limitados dentro de los sistemas rígidos.

El aprendizaje, como función de la logística académica, se deteriora por una alta concentración de actividades académicas en las dimensiones espacio-tiempo, debido a la demanda de los recursos académicos (finanzas, aulas y profesores, computadores, internet, biblioteca, parqueaderos), durante periodos de estudio y la sub-utilización de éstos, en los periodos de restantes. El entorno universitario también experimenta lo que se vive y se hace dentro de los claustros: la cobertura universitaria, el acceso, los costos, las ocupaciones y finalmente el desempeño de los egresados se proyecta en la promoción social, industrial y de la sociedad que lo rodea.

\section{FLEXIBILIZACION DE LA OFERTA ACADÉMICA}

Toda esta problemática que interfiere los sistemas educativos, obligan a repensar la universidad (Salinas, 2000; 2002), haciendo una consideración de la redefinición de sus procesos, principios, políticas, estrategias, organización, operación y gestión (Diaz, 2002), realizando cambios a espacios más amplios y flexibles, enfatizando la necesidad de demoler los muros de rigidez que han estado oprimiendo, modificando la distribución y las formas del ejercicio académico y del poder de las instituciones (Nieto, 2002), reconstruyendo los ámbitos de libertad, responsabilidad, confianza y autonomía, apoyándose en la ciencias y las herramientas tecnológicas actuales, 
influyendo en todas las instituciones la necesidad urgente de crear sociedades de aprendizaje más eficaces y productivas, cambiando culturalmente el contexto del desempeño de modelos rígidos a modelos flexibles y optimizados (Polley, 1997).

Al aprendizaje como el objetivo final de un proceso académico se llega por varias vías: Colectivamente se establecen sociedades de aprendizaje, grupos de trabajo, semilleros de investigación, etc. Individualmente puede darse que un joven sea inteligente o muy inteligente o tiene una buena habilidad para el aprendizaje por lo que requerirá menos esfuerzos, pero el camino normal del aprendizaje se realiza por dedicación, de tal forma que una medida indirecta predictiva del aprendizaje corresponde a la asignación responsable del tiempo que el estudiante le pueda dedicar a un determinado tema. Se establece entonces el concepto de crédito académico (Caspar, 1999) como una unidad estándar de medida del trabajo académico del estudiante, que suma los tiempos de las actividades en la definición de un plan de estudios dentro de un programa académico, que además le dan la oportunidad de ser flexible dentro de unas capacidades funcionales, logísticas y temporales de las instituciones y sus docentes.

En la Fig. 2 se ilustra el diseño del curso académico bajo el concepto de crédito académico cuantificado como el trabajo académico (horas/día) (Castaño, 1993) y toma mayor validez con el planteamiento de una propuesta de flexibilización en la oferta de cursos, es decir la programación de una misma unidad de enseñanza-aprendizaje en un espectro de diversas modalidades de duración, intensidad, inicio y finalización, dando al alumno la posibilidad de seleccionar y organizar su carga académica acorde con situaciones, preferencias, posibilidades y habilidades, involucrando al docente como instructor y acompañante del proceso (Diaz, 2006).

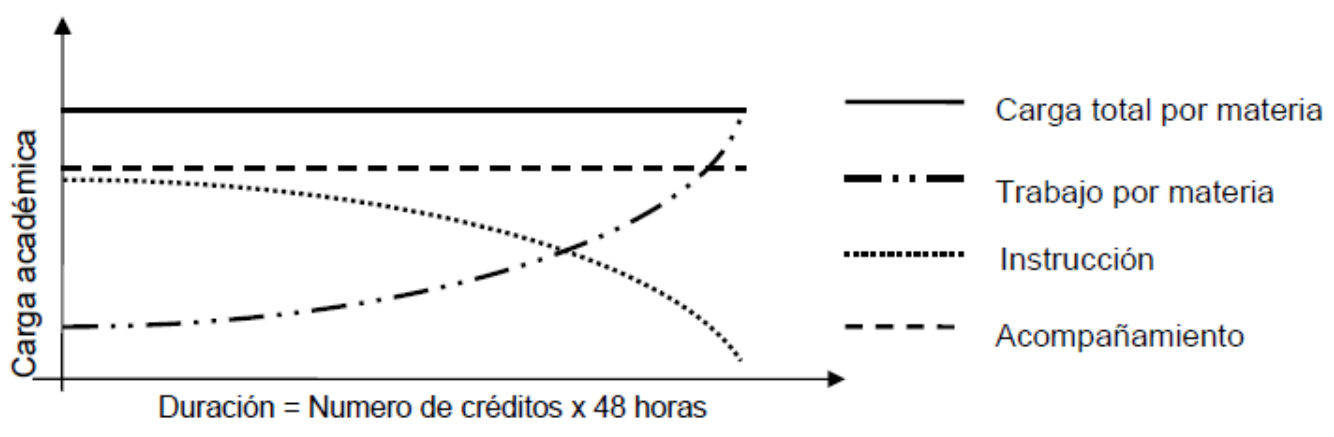

Fig. 2: Distribución de la carga del estudiante de acuerdo al concepto de crédito académico

El estudiante podrá hacer uso de su formación disciplinaria y responsable para configurar su plan de estudios acorde a sus situaciones y su entorno (Salinas, 1997), comprometiéndose más con su crecimiento profesional, eliminando las fronteras temporales entre semestres o periodos cortos y proponiendo nuevas escalas de tiempo de actividades distribuidas e insertadas en el plan de estudios, de tal forma, que la cátedra se valore como un producto con características propias de calidad, de presentación, de oferta y demanda y de beneficios para los estudiantes, profesores y por ende para la misma institución. La flexibilidad en la distribución del tiempo, permite participar de forma integrada a docentes y estudiantes en actividades complementarias de investigación y capacitación, abriendo espacios a la movilidad entendida como la posibilidad para que los estudiantes y los profesores puedan circular a través del sistema académico global (Caspar 1999).

La institución busca mantener su ventaja competitiva y autoridad académica: aumentando su patrimonio básico, "el conocimiento", por medio de programas de capacitación y crecimiento de su planta profesoral y proyectos de investigación, aprovechando las oportunidades de movilidad de los docentes y el conocimiento de los docentes visitantes, renovando sus procesos para un mejor aprovechamiento y acceso de los recursos logísticos, buscando que las inversiones tengan mejor aprovechamiento social y haciendo frente a la dificultad de abatir los costos y mejorar sus niveles de calidad. Una manera de hacerlo es reduciendo los desembolsos por los insumos no benéficos, por ejemplo, el aumento por exceso de la demanda de aulas, libros, computadores, laboratorios, etc., mientras que gran parte del tiempo permanecen sin uso. Otra es la optimización de la planta 
docente y administrativa requerida para generar un eficiente desempeño académico. Estos son factores evidentes que en general, se consideran, pero no reflejan la totalidad de la situación.

\section{MODELO DE APLICACIÓN DE LA FLEXIBILIDAD}

La academia presenta la misma problemática industrial: clientes insatisfechos, productos de mala calidad, entregas a destiempo, productos costosos, etc., donde los criterios generales de calidad se miden a la salida del proceso (Casassus, 1999). Los modelos que son exitosos en el ambiente industrial aplican también a la academia, dado que el conocimiento es un producto con características idénticas a cualquier producto industrial, con pretensiones de altos niveles de calidad, entendiéndose todo el contexto sociológico que la universidad y la academia en general implica, desde el mismo momento de la selección de los aspirantes al programa universitario, su permanencia y finalmente su evaluación y desempeño en la sociedad con resultados finales en el momento que el estudiante logra ser un egresado, o con resultados parciales al finalizar un periodo o una asignatura.

La propuesta de flexibilización de la oferta académica (Diaz, 2006), se ajusta a la filosofía estratégica del Justo a Tiempo (JIT) (Palacios y Tomas, 1993), utilizada con mucho éxito en manufactura, donde los postulados objetivos trasmiten la responsabilidad al usuario final, pregonando que se debe darle al cliente (aprendiz) lo que él quiere, como lo quiere y cuando lo quiere, eliminando desperdicios tales como tiempo, recursos logísticas y recursos académicos, reduciendo así los costos de la educación y por otro lado mejorando la calidad del proceso de enseñanza aprendizaje, como parte de la tendencia mundial en esa dirección. El propósito fundamental es optimizar acordemente los recursos en beneficio de los estudiantes, profesores y la universidad, buscando que cada estudiante establezca su ritmo de estudio según sus capacidades, actividades y entorno social.

Entendiendo que cada estudiante es un individuo con características particulares, pero que pueden ser asociados por espectros de similitud y tiempo, el JIT permite administrar las actividades por comunidades con características más homogéneas, haciendo que se puedan desarrollar programas de moda para poblaciones numerosas, ofreciendo productos académicos en varias presentaciones de temáticas, modalidad, intensidad y tiempo de la oferta. En este sentido muchas universidades han reformado su pensum con contenidos más flexibles, donde una adecuada orientación permite al estudiante ser más especializado en cierta área del conocimiento. Para ilustrar esta situación, se plantea un ejemplo de oferta de cursos en una universidad donde se atienden clases por grupos pequeños del orden de 40 estudiantes, se entendería que para una población de 1000 estudiantes se deben programar 25 grupos, los cuales en un modelo rígido se ofrecen en forma paralela y simultánea durante el periodo académico. En el modelo de oferta flexible de acuerdo a la demanda, se pueden definir varias presentaciones de estos cursos. En las tablas 1 y 2 , se ilustran algunas alternativas de clasificación de estudiantes:

Tabla 1: Clasificación de oferta de cursos por temática o modalidad de grupos de estudiantes

\begin{tabular}{|l|l|}
\hline N1 & Estudiantes que repiten el curso, base no nula \\
\hline N2 & Estudiantes que tienen interés normal en el curso \\
\hline N3 & Estudiantes que tienen mucho interés en el curso \\
\hline N4 & Estudiantes que tienen dificultades de entorno (trabajan, etc) \\
\hline
\end{tabular}

Esta primera clasificación, puede inducir a redefinir la duración total del curso, desde una presentación básica, hasta una presentación de énfasis en forma más profunda e intensiva. La segunda modalidad permite definir las presentaciones de los cursos de acuerdo a la intensidad y duración definida en el concepto de crédito académico. Naturalmente que el sistema JIT no es lo único que necesita una universidad para competir, pero es ya evidente que nadie seguirá siendo competitivo por mucho tiempo sin que se asuman los comportamientos y las posibilidades de avance que dicho sistema ofrece (Lefcovich, 2004). 
Tabla 2: Clasificación de oferta cursos por intensidad y duración

\begin{tabular}{|l|l|}
\hline N5 & Intensivo, curso remedial (periodo corto de refuerzo) \\
\hline N6 & Intensivo Normal (duración en semanas = Número de créditos) \\
\hline N7 & Dedicación de medio tiempo (duración en semanas = 2 x Número de créditos) \\
\hline N8 & Dedicación un cuarto de tiempo (duración en semanas = 4 x Número de créditos) \\
\hline N8 & Extendido (duración más allá del periodo académico) \\
\hline
\end{tabular}

Al mirar el concepto de la propuesta desde la misión de la Universidad y desde el punto de vista de la formación de un profesional íntegro, la flexibilización actúa sobre las posiciones y disposiciones (Diaz, 2002) que cubren el desarrollo de diversas estrategias que fomentan el sentido de responsabilidad y la autonomía en la búsqueda del conocimiento. Es necesario considerar que la propuesta de flexibilidad de la oferta académica tiene el objeto de calidad sobre cada uno de los estamentos involucrados en ella. Por lo tanto, tiene una cantidad de implicaciones, que le dan fortaleza al sistema académico a favor de los estudiantes, los docentes y la misma institución. Muchas instituciones buscan una colección de certificaciones por dependencias y hasta de la institución completa, pero en el fondo no engranan en el enfoque sistémico, dejando por fuera lo fundamental y la razón de ser de la institución respecto a la oferta y al desempeño académico. Toda esta actividad gira en torno a factores culturales, psicológicos, económicos, problemáticos, logísticas, académicos, de proyección social, entre otros, que en buena parte intervienen en el proceso de aprendizaje de los estudiantes. La elaboración de una estrategia competitiva supone definir aquellas variables objetivas en las cuales se quiere ser superior a la competencia. En la academia se diría de aquellas variables que permiten estar acreditados y que hacen que más estudiantes se matriculen en la institución. Se pueden enumerar cinco variables que servirán de base para conseguir esa ventaja competitiva: costo, calidad, servicio, flexibilidad e innovación (Lefcovich, 2004).

Cada curso se define dentro de unos modelos pedagógicos propios de acuerdo con sus características de modalidad, de intensidad, duración y relación con otros, entonces la transformación metodológica debe ser propia para cada uno de los cursos ofrecidos, teniendo en cuenta su grado de dificultad, el tiempo necesario para la asimilación de los contenidos o tardanza de resultados, seguimientos, su grado de "mortandad" y sus procesos evaluativos, dando diferentes opciones de elección según las habilidades, deseos, necesidades y preferencias de cada estudiante, siendo necesario definir las características de oferta, además de las características propias: número de créditos, intensidad horaria semanal, presencial y no presencial, intensidad horaria semestral, co-requisitos, pre-requisitos, modelos pedagógicos, recursos académicos necesarios, características del docente, código, fecha de inicio y finalización de cada curso, intensidad horaria semanal, aulas donde se desarrollaran los cursos dependiendo directamente de la funcionalidad (laboratorio o aula de clase presencial o virtual, biblioteca) y el ritmo de estudio de cada estudiante.

La primera impresión que tienen las directivas universitarias, está relacionada con los costos. Pero la experiencia de la flexibilidad y el JIT en los sistemas industriales, han demostrado que es verdad que el ahorro es fundamental y su estrategia es la eliminación de los despilfarros. En un sistema de oferta flexible se juega con la disponibilidad de recursos pedagógicos, logísticos y humanos, permitiendo mayores espacios y una mayor rotación de los estudiantes, haciendo que los procesos masivos se realicen en forma más continua y más distribuida, evitando así las congestiones en los diversos sistemas y por consiguiente su mayor aprovechamiento y aumento de calidad a un costo adecuado.

Universalizar las características de las personas, cuando estas son individuos, es un grave error, no pudiendo aplicarse las mismas metodologías a toda una población. En tal sentido los esfuerzos pedagógicos se esfuerzan en pensar en el estudiante construyendo metodologías centradas en él, particularizando factores como: concentración, memorización, tranquilidad, comprensión, también el dominio personal de habilidades y técnicas de estudio y estrategias de aprendizaje, variables situacionales como actividades, dificultades, herramientas, etc. La flexibilidad de oferta propicia un ambiente de aprendizaje distribuido más eficientemente en el tiempo, ayuda a potencializar estos 
factores, incluyendo componentes de educación a distancia (Salinas, 1998), educación abierta e inclusive clases en tiempo real. También es un error pensar que la flexibilidad puede con todo, lo flexible también es limitado; de ahí que no se puede pensar en solucionar de forma flexible cada caso de estudiante en forma particular. Entonces el nivel de flexibilidad será el resultado de un proceso de optimización donde se equilibre la masificación, los beneficios y los costos. Es cierto el interés de los gobiernos de nuestros países en mirar el aumento de la cobertura y la disminución en la deserción universitaria; también es cierto que esta meta tiene costos y requiere de inversiones (Guerra et al., 2002). El concepto de una oferta flexible permite manejar los periodos de altas demandas y promocionar los periodos más flojos, de tal forma que el desempeño institucional, se realice en forma holgada y por consiguiente la capacidad logística instalada podrá ser utilizada con un mayor volumen de estudiantes, aumentando el nivel de aprovechamiento de los recursos.

El costo del modelo tradicional para aumentar la capacidad de cobertura está relacionado con el presupuesto que las instituciones deben realizar adicionalmente a su logística ya existente, además del aumento de su planta docente. Grosso modo, se podría decir que si va a crecer la cobertura en un $25 \%$, la planta docente debería aumentar en un $25 \%$, porque la carga actual del docente ya es suficiente y en este aspecto hay que invertir en recurso humano. Los costos logísticos resultan en beneficio por el aprovechamiento de los tiempos de no utilización. Por ejemplo un libro de biblioteca en el modelo rígido, es demandado masivamente en pocos días previos a la evaluaciones, en un modelo flexible se tendrán muchos grupos pequeños distribuidos en todo el periodo, haciendo uso continuo con mejor aprovechamiento y menor demanda masiva.

En muchos países los gobiernos financian las universidades públicas, pero las universidades privadas no tienen financiamiento en los rubros de formación pregrado, el cual es asumido directamente por ingresos de las matrículas de los estudiantes. Este rubro es costoso para una clase media y baja, la cual cubre gastos de formación y el derrame de los gastos logísticos que generalmente tienen componentes de inversión a largo plazo. En un modelo de oferta flexible, los gastos de matrícula pueden también ser distribuidos de acuerdo con el mercadeo de los cursos, beneficiando a los estudiantes de bajos recursos, obteniéndose para la universidad un flujo más continuo de ingresos.

\section{OPERATIVIDAD DE LA OFERTA FLEXIBLE}

Partiendo de la definición de crédito académico (figura 2), la carga académica total del estudiante es la suma de las cargas de todos los cursos, la cual se busca mantenerla constante durante el transcurso del periodo académico. Para mayor flexibilidad se puede jugar con la planeación de cursos en periodos más largos, por ejemplo periodos anuales de 40 a 46 semanas académicas, tal como se programa las ofertas de cursos en educación continua, especializaciones, maestrías, doctorados o como lo hacen algunas instituciones de capacitación no académicas: institutos de idiomas, artes, oficios, etc.

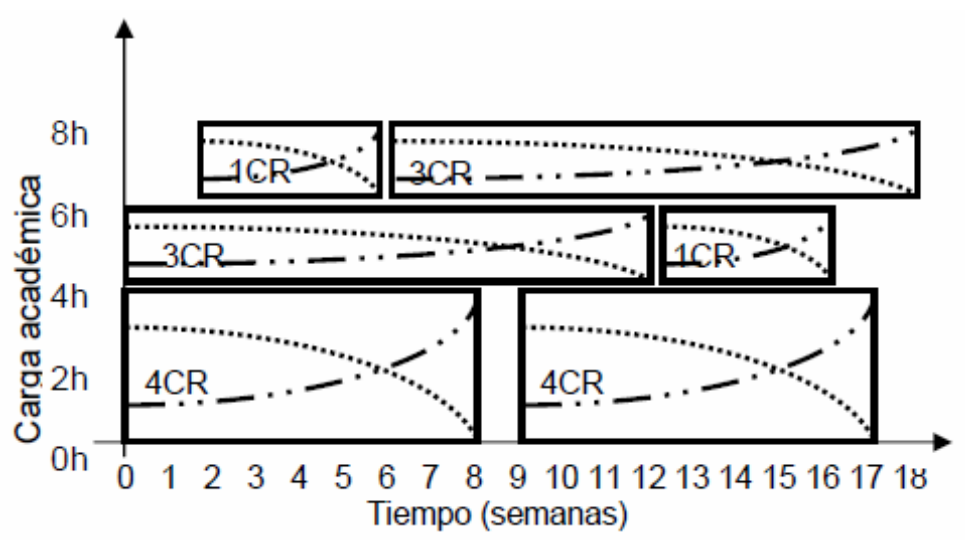

Fig. 3: Ejemplo de distribución flexible de carga académica (seis materias), en un periodo semestral de 18 semanas para 16 créditos y un equivalente de 2 créditos de holgura o descanso. 
En la figura 3, se presenta un ejemplo de distribución de carga académica aplicada a un periodo de 18 semanas (modelo semestral Colombiano), con cursos más intensivos y cortos, insertados con posibles pequeños periodos de holgura o descanso. Se puede apreciar que la dedicación al trabajo académico por el estudiante es más distribuida, remediándose el problema del acoso al fin de periodo por mayor demanda de trabajo académico y simultaneidad en la presentación de evaluaciones. Similarmente la gestión académica deja de ser masiva y se vuelve más fluida, con la posibilidad de una mejor atención de los procesos, dando lugar a la minimización de errores. Los posibles fallos pedagógicos afectaran solo poblaciones pequeñas de estudiantes, de los cuales se tendrá una respuesta rápida para realimentar las correcciones a grupos posteriores, con la ventaja de tener tiempo para realizarlas oportunamente. Por su parte el docente también experimenta los beneficios de la distribución de la oferta, al atender menos grupos simultáneos pero con mayor dedicación en periodos más cortos de tiempo. Es de anotar que la suma total de estudiantes atendidos en el periodo académico sigue siendo igual; lo diferente es la forma de atención y el acompañamiento más fluido.

\section{CONCLUSIONES}

La mayor dificultad en el sistema académico rígido es considerar el volumen del aprendizaje sin detenerse a reflexionar en la individualidad del estudiante y su incidencia en la calidad del aprendizaje, con métodos de acoso académico por saturación de actividades, sobre todo en momentos que la institución ordena su realización masiva y simultánea, convirtiéndose en un problema de cuello de botella en el desempeño del estudiante, donde la única alternativa resultante es el bajo nivel de calidad del aprendizaje y la congestión de los procesos académicos. A su vez la aplicación del concepto JIT a la academia, permite definir el producto académico similarmente a un producto manufacturado con características de producción, oferta, mercadeo, utilización y calidad, pudiéndose aplicar los mismos conceptos industriales para la oferta de un producto de calidad, donde la variable operativa de oferta corresponde al nivel de flexibilidad. La oferta académica flexible es la alternativa estratégica operativa en la educación superior, para hacer de la enseñanza y el aprendizaje del conocimiento un servicio de calidad para la satisfacción del estudiante, el mejor desempeño docente y el mejor servicio de la administración, ligando la actividad académica al desarrollo del hombre y la sociedad, movilizando las estructuras de enseñanza, aprendizaje e investigación en beneficio de toda la comunidad, ajustándose continuamente a las exigencias de la demanda diversificada de la población estudiantil.

\section{REFERENCIAS}

Carbajo, M.; Causas Sociales e intelectuales de la Revolución Francesa, Coro de Babel (2006), http://www.corodebabel.com.ar/index2.php?option=com_content\&do_pdf=1\&id=19. Octubre (2007).

Caspar, E.; The Bologna Declaration of 19 June 1999; The European higher education area, Bologna (1999), http://www.bologna-berlin2003.de/pdf/bologna_declaration.pdf, Acceso: Octubre (2007).

Casassus, J.; Lenguaje, Poder y Calidad de la educación, Proyecto principal de educación en América latina y el Caribe, N050, Pág. 47, Santiago-Chile (Diciembre, 1999). http://unesdoc.unesco. org/images/0011/001191/119117s.pdf\#119121. Acceso: Octubre (2007).

Casassus, J.; Problemas de la gestión educativa en América Latina (la tensión entre los paradigmas de tipo $A$ y el tipo B, UNESCO (2000), http://www.unesco.cl/medios/biblioteca/documentos/gestion_

problemas_gestion_educativa_casassus.pdf. Acceso Octubre (2007).

Castaño, J.M.; Marketing justo a tiempo, Ed. Norma, ISBN: 958-04-2114-5, pp 39-68, Bogotá (1993).

Diaz, A.G.; La flexibilización de la oferta académica mejora la calidad del aprendizaje; RIE, ISSN: 1681-5653, (en línea), N039/5, Octubre 2006, http://www.rieoei.org/1332.htm. Acceso Oct. (2007).

Diaz, V.M.: Flexibilidad de la Educación Superior en Colombia: Proyecto Estándares Mínimos de 
Calidad, ICFES (Instituto Colombiano para el Fomento de la Educación Superior), Bogotá D.C., (2002), http://www.icfes.gov.co/cont/s_fom/pub/libros/libro flexibilidad y educacion.pdf.

Delors, J.; La Educación encierra un Tesoro, Santillana Ediciones UNESCO, ISBN 92-3-303274-4, pp 9 30, Madrid (1996).

Guerra, M.R., N. Pabón y J.M. Restrepo; Flexibilidad curricular: mayor equidad en el acceso y permanencia en la educación superior, Revista de la Educación Superior (ANUIES), ISSN: 0185-2760 (en línea), XXXI(123), Julio-Septiembre, 2002. http://www.anuies.mx/servicios/p_anuies/ publicaciones/revsup/res123/art8.htm, Acceso: Noviembre (2007).

Kant, I.; Pedagogía, http://psikolibro.webcindario.com/libros/ccss/Immanuel Kant - Pedagogia.zip, 1803, Acceso: Octubre (2007).

Lefcovich, M.; Producción justo a tiempo para la competitividad total, http://www.navactiva.com/ web/es/alog/doc/informes/2004/10/28464.php (2004), Acceso: Noviembre (2007).

Leite, A.; Representaciones sobre el éxito y fracaso académico en estudiantes universitarios. Aproximaciones teóricas y empíricas, Primer Congreso Nacional de Investigación Educativa. Univ. Nacional del Comahue, Fac. de Ciencias de la Educación. Octubre de 1999.http://www.unne.edu.ar/ Web/cyt/cyt/humanidades/h-028.pdf, Acceso: Octubre (2007).

McMeekein, R.W.; La investigación al servicio de la educación: Tiempo y aprendizaje, CIDE/REDUC, Boletín 30, Abril (1993).

Nieto, L., La flexibilidad curricular en la educación superior, Algunas perspectivas para su análisis, México, (2002); http:/ambiental.uaslp.mx/docs/LMNC-PN-0210-flexcurr.pdf., Acceso: Octubre (2007).

Palacios, B. y M. Tomás; El sistema Just In Time y la flexibilidad de la producción, Editorial Pirámide. ISBN: 84-368-0731-6, 263 pp, Madrid (1993).

Ohno, T., Toyota Production System, Productivity Press, ISBN: 978-0-915299-14-0, pp 144 , Toyko, Japon, (1988).

Polley, A.; What Will Transformation Mean for Traditional Universities?, Technology and Learning; virginia.edu Volume I, Number 2; (1997); http://www.itc.virginia.edu/virginia.edu/ fall97/trans/all.html, Acceso: Octubre (2007).

Pedroza-Flores, R.: La Flexibilidad Académica en la Universidad Pública, Revista de la Educación Superior (ANUIES), ISSN: 0185-2760 (en línea), XXX(119), Julio-Septiembre, 2001. http://www. anuies.mx/servicios/p_anuies/publicaciones/revsup/res119/art7.htm.

Salinas, J.; Enseñanza flexible, aprendizaje abierto. Las redes como herramientas para la formación, Edutec, n¹0, 02/99. (1997)

Salinas, J., ¿Qué se entiende por una institución de educación superior flexible?, En: Cabero, J; Salinas, J. (coord.). Las nuevas tecnologías para la mejora educativa. Kronos. Sevilla. ISBN: 8485101-25-1, pp. 451-466 (2000), http://gte.uib.es/articulo/edutec991.pdf., Acceso: Octubre (2007).

Salinas, J.; Nuevos ambientes de aprendizaje para una sociedad de la información; Revista Pensamiento Educativo. PUC Chile. 20, 81-104 (1997).

Salinas J.; Redes y educación: Tendencias en educación flexible y a distancia, II Congreso Internacional de Comunicación, tecnología y educación. Oviedo. pp. 141a151. (1998), http://www.uib.es/depart/gte/ tendencias.html., Acceso: Octubre (2007).

Salinas, J.; Modelos flexibles como respuestas de las universidades a la sociedad de la información, Acción Pedagógica, 11 (1), 4-13 (2002), Universidad de los Andes, http://www.comunidadandina.org/ bda/docs/VE-EDU-0010.pdf. Acceso Noviembre (2007).

UNESCO; Conferencia Mundial sobre Educación Superior, Paris, (5 a 9 de octubre de 1998), http://unesdoc.unesco.org/images/0011/001173/117363s.pdf., Acceso: Noviembre (2007). 